[2] OrLa-Jensen. Moelkepasteuriseringsspörgsmaalet i moderne Belysning Mólkeritidende, 1916.

[3] Orta-Jensen. De l'origine des oxydases et réductases du lait de vache. Revue générale du lait, 1906.

[4] Schardinger. Ueber das Verhalten der Kuhmilch gegen Methylenblau. Zeitschrift für Untersuchung der Nahrungs- und Genussmitteln, 1902.

[5] SCHERN K., Ueberdie Schardinger Reaktion der Milch. Biochem. Zeitschrift, 1909.

[6] OrLa-Jensen. Mrelkeri-Bakteriologi.

[7] LIND C. Reduktasepröven sammenlignet med Bakterie.Tcelningsmetoden. Mcelkeritidende, 1915.

[8] BaRthel Chr. Fortsatta Undersökningar rörande Reduktasprovet. Meddelande - Nr. 141 fran Centralanstalten f. försöksväsendet pa jordbruksomradet, 1917.

[9] OrLa-JENSEn, De l'action du chauffage sur le lait de vache. Revue générale du lait, 1905.

[10[ WEBER. Die zur Unterscheidung roher und gekochter Milch dienenden Untersuchungsmetoden. Diss. Leipzig, 1902.

[11] BUttenberger. Zur Unterscheidung der pasteurisierten Milch. Zeitschrift f. Unters, der Nahrungs- u. Genussmitteln, 1906.

[12] Jensen C. O. Moelk og Moelkekontrol, 1903.

[13] Sommerfeld. Handbuch der Milehkunde, 1909.

\title{
RECHERCHES SUR L'INFLUENCE DE LA SÉCRÉTION LACTÉE SUR L'ORGANISIVIE, LE SYNDROME HYPOGLYCÉMIQUE ET LA FIËVRE VITULAIRE DE LA VACHE
}

\author{
par M. L. AUGER \\ Docteur-Vétérinaire, Chef de travaux de clinique à l'Ecole vétérinaire de Lyon
} (Suite et fin)

Le syndrome hypoglycémique obtenu expérimentalement chez la vache se traduit par des symptômes identiques à ceux de la fièvre vitulaire. - Si nos idées sont exactes et si la fièvre vitulaire est la conséquence d'un épuisement du glucose immédiatement utilisable de l'organisme, on doit pouvoir en créant artificiellement cet état chez une vache saine reproduire tous les signes de la fièvre vitulaire. L'épuisement de l'organisme en glucose immédiatement utilisable peut être décelé par l'abaissement du taux du sucre du sang, mais il se traduit aussi par des symptômes particuliers dont l'ensemble constitue ce que l'on appelle aujourd'hui le «syndrome hypoglycémique ». A la vérité ce syndrôme avait pu être obtenu autrefois par l'injection d'une substance provoquant de la glycosurie : la phloridzine dont le mode d'action est d'ailleurs encore discuté et l'ensemble des signes présentés par les animaux était connu sous le nom d'"intoxication glycoprive", mais les résultats donnés par la phloridzine étaient inconstants et e'est surtout depuis l'extraction de l'insuline par les Canadiens qu'une telle expérimentation est devenue facile. 
Pour que les accidents hypolycémiques apparaissent, il faut que le taux du sucre sanguin s'abaisse à un niveau donné, lequel varie quelque peu dans chaque espèce et avec chaque sujet. Chez les animaux de l'espèce bovine, en prenant des chiffres moyens, il faut que la glycémie s'abaisse au dessous de 0 gr. 40 au litre, jusqu'à 0 gr. 30 et moins pour observer les accidents qui constituent le syndrome hypoglycémique. Ces déductions étaient admises jusqu'à ces derniers temps; de nombreuses expériences montrent qu'elles ne sont pas toujours exactes. Le sang contient, nous venons de le voir, une très faible quantité de glucose qui chez les sujets en bonne santé reste à peu près fixe. Les organes puisent dans le sang le glucose nécessaire à leur fonctionnement : travail musculaire, élaboration du lactose du lait etc., et le sang lui-même pour maintenir normal et à peu près fixe le taux de son glucose puise à son tour dans les réserves hydrocarbonées de l'organisme, en l'espèce le glycogène. Lorsque l'alimentation est peu riche en glucose, l'organisme peut en fabriquer aux dépens des albumines et des graisses, mais cette élaboration n'est pis immédiate, elle demande un certain temps. Lorsque la consommation du glucose est considérable et rapide, on observe bien une chute de la glycémie régulière au début dans beaucoup de cas, et le syndrome hypoglycémique peut apparaître, mais alors qu'il dure, la giycémie ne reste pas uniformément basse, des apports glucosés provenant des réserves glyeogéniques de l'organisme arrivent au sang, la glycémie s'élève, puis la consommation de glucose étant toujours grande, elle s'abaisse de nouveau, en d'autres termes on observe au lieu d'une hypoglycémie constante, des oscillations souvent très marquées de la glycémie traduisant un trouble profond de la glyco-régulation. C'est ce qui nous explique les constatations d'un grand nombre d'auteurs qui ont vu le syndrome hypoglycémique apparaître avec un taux normal du glucose dans le sang, parfois même avec de l'hyperglycémie, suivant le moment où le prélèvement du sang pour le dosage a été pratiqué. Ces faits ont été signalés non seulement avec l'insuline, mais avec des substances analogues retirées des tissus animaux autres que le pancréas et des tissus végétaux et qui sont connues sous le nom d'"insulinoïdes ". Il y a là un phénomène encore obscur dans le métabolisme des hydrates de carbone; le glucose sanguin circulant n'est-il pas directement utilisable par les tissus comme on l'a prétendu, doit-il subir certaines transformations ? On est encore mal fixé sur ce point. Certains auteurs avaient même prétendu que le syndrome hypoglycémique obtenu par l'injection d'insuline qui se traduit, nous allons le voir, par des phénomènes de coma et de convulsions, n'était pas provoqué par l'épuisement du glucose de l'organisme, mais par des substances toxiques qui pouvaient être contenues dans l'insuline. Cette interprétation n'est pas exacte, parce que. si on injecte à la fois du glucose et de l'insuline, aucun trouble n'apparâ̂t et, d'autre part, l'injection d'une quantité convenable de glucose 
guérit le syndrome hypoglycémique. D'ailleurs, lorsque des animaux ont succombé au coma hypoglycémique, si l'on pratique le dosage du glycogène du foie et des muscles, qui sont les organes qui normalement en contiennentle plus, on constate que cette substance a disparu à peu près complètement. Linsuline fait donc disparaître non seulement le glueose sanguin, mais épuise aussi les réserves hydrocarbonées de l'organisme.

Retenons, pour le moment, qu'une injection d'insuline lorsqu'elle est suffisante fait apparaitre des symptômes spéciaux, toujours les mêmes : coma et convulsions qui constituent le syndrome hypoglycémique et que le dosage du glucose sanguin pendant toute la durée de oes symptômes donne des résultats variables traduisant un trouble profond de la glyco-régulation, à certains moments et le plus souvent de l'hypoglycémie, ou bien une glycémie normale, voire même de l'hyperglycémie.

On ne provoque pas à coup sûr le syndrome hypoglycémique en injectant à un animal une dose donnée d'insuline proportionnelle à son poids. De nombreux facteurs interviennent qui favorisent ou entravent l'action de l'insuline; parmi ceux qui nous intéressent particulièrement nous citerons: l'alimentation antérieure, la lactation et l'intégrité des organes qui règlent le eycle des hydrates de carbone dans l'organisme.

L'alimentation antérieure est un facteur important. Elle permet, lorsqu'elle est abondante, l'accumulation de réserves dans l'organisme : hydrates de carbone, graisses, albumine et la physiologie a démontré les transformations dans l'organisme, suivant ses besoins, d'albumine et de graisse en hydrate de carbone. Tous les expérimentateurs qui se sont occupés de l'action de l'insuline ont noté l'importance de ce facteur ; e'est ainsi que Penau et Simonnet, pour nous borner à une citation, ont vu que les lapins nourris à l'avoine étaient beaucoup plus résistants à l'insuline que ceux nourris au foin, parce que cet aliment plus alibile est une meilleure source de glycogène. Nous avons fait remarquer aussi au chapitre précédent que la glycémie est toujours plus élevée chez les vaches qui reçoivent des aliments concentrés que chez celles qui n'en consomment pas; nous avons vu également que les éleveurs anglais avaient remarqué que l'on observait rarement la fièvre vitulaire sur des vaches fortement nourries dans les semaines qui précédaient le vêlage, alors que leur rendement en lait était encore augmenté et qu'ils avaient érigé cette constatation en méthode zootechnique sous le nom de "steaming " ou " mise sous pression ". Toutes ces constatations se corroborent et se complètent et sont des arguments en faveur de la théorie de l'hypoglycémie dans la fièvre vitulaire.

De même qu'une lactation abondante abaisse la glycémie, arrive à troubler la glyco-régulation et favorise au premier chef l'apparition de la fièvre vitulaire, de même elle rend les animaux plus sensibles à l'insuline, vraisemblablement parce que les réserves hydrocarbonées et autres sont minimes chez les vaches grandes laitières qui doivent 
incessamment fournir une grande quantité de matériaux pour l'élaboration du lait. En fait, bien qu'à la suite d'une injection d'insuline, la sécrétion lactée soit partiellement tarie, il faut moins d'insuline pour provoquer des accidents chez une vache laitière que sur un animal de boucherie. Nous avons tenté de démontrer qu'il se produit toujours au moment où s'établit la sécrétion lactée une chute de la glycémie, mais si nous avons dit que nos expériences à ce sujet étaient contestables, parce que dans les dosages pratiqués avant l'accouchement, du lactose, provenant de la rétention lactée, au moment où s'élabore le colostrum, lait de rétention, pouvait s'ajouter au glucose du sang, nous avons toujours eonstaté, en revanche, que c'est à cé moment que les animaux sont les plus sensibles à l'insuline. Des vaches, des chèvres, des chiennes ont reçu avant l'accouchement une dose donnée d'insuline sans présenter d'accidents, alors qu'un ou deux jours après le part, au moment où s'établissait la sécrétion lactée, la même dose d'insuline faisait apparaître le syndrôme hypoglycémique.

Rappelons que c'est à ce moment-là aussi que le plus souvent apparaît la fièvre vitulaire.

Un grand nombre d'organes ou de glandes interviennent dans le métabolisme des hydrates de carbone : foie, reins, surrénales, thyroïde, etc., et il faut bien reconnaître que le rôle dévolu à chacun d'un est loin d'être connu, mais celui du foie et des reins paraît primordial. Nous avons constaté à plusieurs reprises que les animaux qui présentent des lésions rénales et hépatiques sont plus sensibles à l'insuline que les sujets normaux; la glyco-régulation est imparfaite chez eux et toute substance, comme l'insuline, qui tend à la troubler momentanément, a des effets plus marqués.

On peut rapprocher de ces constatations les faits suivants : certaines vaches présentent des accès de fièvre vitulaire presque à chaque vêlage et malgré le traitement d'Evers elles finissent pas succomber; or, à l'autopsie de ces animaux on a fréquemment trouvé des lésions hépatiques (Delamarre-Maguire). Si la fièvre vitulaire est, comme nous le pensons, un trouble passager de la glyco-régulation on conçoit qu'elle apparaisse plus facilement chez ces sujets où les organes qui règlent le cycle des hydrates de carbone sont touchés. C'est là un facteur très important sur le rôle duquel il est nécessaire d'insister. La fièvre vitulaire le plus souvent apparaît sur des vaches en bonne santé, à la suite de la mise en train de la mamelle; sous l'influence de la spécialisation zootechnique cette glande anormalement développée demande trop, il lui faut plus de glucose que n'en peut fournir l'organisme pour l'élaboration du lactose, la glyco-régulation est faussée, l'hypoglycémie etla maladie apparaissent; ces troubles disparaissent rapidement par l'insufflation de la mamelle, qui, nous le verrons plus loin, annihile seulement d'une façon toute mécanique le fonctionnement de cette glande. Lorsque le foie ou les reins 
sont lésés, non seulement la maladie apparaît plus facilement, plus souvent, mais encore la glyeo-régulation étant troublée d'une façon permanente, les animaux guérissent difficilement par le traitement d'Evers; il faut faire deux ou trois insufflations et le retour à la santé parfaite demande plusieurs jours; ce sont le plus souvent ces sujets qui succombent à l'accident. Ces constatations ont encore un autre intérêt; elles permettent de rattacher la fièvre vitulaire, le plus souvent simple trouble de la glyco-régulation survenant chez des femelles en bonne santé, mais spécialisées en vue de la production du lait, d'autres fois trouble de la glyco-régulation sous la dépendance à la fois de la spécialisation zootechniqueetde lésions organiques, àl'éclampsiedesautres espèces, maladie avec laquelle cliniquement elle a les plus grandes analogies et où les lésions hépatiques et rénales jouent un rôle de premier plan.

Quoiqu'il en soit, nous avons pu provoquer le syndrôme hypoglycémique chez la vache laitière par l'injection de fortes doses d'insuline; les symptômes qui le earactérisent sont identiques à ceux de la fièvre vitulaire : somnolence, torpeur, coma, chute de la température ; certaines attitudes, incurvation de la tête en décubitus sternal ou en décubitus latéral complet que l'on observe souvent au cours de la fièvre vitulaire, se rencontrent aussi dans le syndrôme hypoglucémique.

Les deux photographies d'une vache en plein coma hypoglycémique que nous donnons ci-dessous reproduisent ces curieuses attitudes qui sont d'observation courante au cours de la fièvre vitulaire.

Après l'injeetion d'insuline, les animaux dans $50 \%$ des cas reviennent d'eux-mêmes à la santé, l'action de eette substance s'épuisant avant les réserves hydrocarbonées de l'organisme; dans la fièvre vitulaire, on ne perdait d'ailleurs avant le traitement d'Evers que $50 \%$ des animaux. D'autres fois, une injection glucosée est nécessaire, elle amène la guérison en quelques minutes si elle est faite peu de temps après le début des accidents. Mais lorsque le sujet est en plein coma, il faut une demi-heure, quelquefois davantage pour que la guérison survienne. Dans certains cas même, l'injection glucosée est sans effet, on peut la répéter à plusieurs reprises, le coma entrecoupé de convulsions ne cesse pas et les malades succombent. A l'autopsie de ces sujets, nous n'avons pas rencontré de lésions récentes, mais des lésions anciennes du foie et des reins. De même certaines vaches atteintes de fièvre vitulaire succombent malgré l'insufflation mammaire, malgré une injection glucosée et à l'autopsie de ces animaux on rencontre souvent des lésions hépatiques : lésions de dégénérescence graisseuse le plus souvent. Cela nous montre encore l'importance du facteur : intégrité de certains organes, sur lequel nous venons d'insister.

L'insufflation des mamelles amène une augmentation du taux du glucose du sang. - Il nous reste à expliquer les effets du traitement d'Evers, lequel consiste dans l'insufflation sous pression de la 
mamelle. De nombreuses hypothèses ont été émises à ce sujet. Au début, on injectait une solution d'iodure de potassium dans la mamelle, pour, pensait-on, neutraliser les poisons qui s'y trouvaient contenus, puis on vit par hasard que l'injection d'air produisait les mêmes effets ; on pouvait encore croire que l'air agissait par l'oxygène qu'il contient, mais on ne tarda pas à se rendre compte que l'injection d'un gaz inerte, de l'azote, par exemple, d'un liquide quelconque, de l'eau bouillie amenait la guérison, à condition que cette injection fut faite sous pression. C'est ce qui a fait dire à RoBIN, avec juste raison d'ailleurs, qu'il était vraisemblable que le traitement d'Evers agissait d'une façon toute mécanique. On a pensé aussi que l'insufflation mammaire relevait la pression sanguine qui serait fortement diminuée au cours de la fièvre vitulaire, soit en refoulant le sang de la mamelle dans la grande circulation, soit par action réflexe. II n'en est rien. En collaboration avec le professeur JuNG, nous avons montré au moyen de tracés de pression pris à l'intérieur de la carotide chez des vaches et des chèvres que l'insufflation mammaire était sans action sur la pression sanguine. Comment agit donc le traitement d'Evers? Vraisemblablement en supprimant par la distension des acini glandulaires par l'air sous pression, le fonctionnement de la mamelle. Lors de ses recherches sur l'origine du lactose, le professeur Ch. Porcher pratiquait l'ablation des mamelles chez les vaches et les chèvres, soit avant l'accouchement, soit au cours de la lactation et il constatait après le part, du moment où devait s'établir la sécrétion lactée dans le premier cas, immédiatement après l'opération dans le second, une augmentation du glucose-du sang proportionnelle au rendement en lait de la femmlle opéré, Le glucose fourni par l'organisme pour l'élaboration du lactose n'était plus utilisé, il s'accumulait dans le sang, puis ne tardait pas à atteindre le seuil et était éliminé par les urines. Après l'insufflation mammaire, on fait exactement les mêmes constatations qu'après l'ablation des. mamelles ; on observe toujours une élévation du taux du glucose sanguin et une glycosurie en rapport direct avec le rendement en lait de l'animal en expérience. Ces faits sont démontrés par de nombreuses recherches effectuées par WIDMARK et CARLENs et nous-même. En un mot, l'insufflation équivaut à une "ablation temporaire des mamelles ", si je puis employer cette expression, à l'annihilation complète, mais momentanée de leur fonctionnement. Un vétérinaire américain Menig, en liant les artères mammaires, dans la fièvre vitulaire, et en empêchant ainsi l'absorption du glucose du sang par la mamelle a obtenu neuf fois la guérison sur neuf cas traités et aussi rapidement que par l'insufflation mammaire. Ces faits sont un argument d'un certain poids pour la démonstration de l'existence de l'hypoglycémie au cours de la fièvre vitulaire.

L'hypoglycémie au cours de la fièvre vitulaire. - A première vue, si nos idées sont exactes, il semblerait que pour apporter la dernière 
pierre à l'édifice et démontrer que la fièvre vitulaire est bien la conséquence de l'hypoglycémie, il n'y ait qu'à doser le sucre du sang sur des vaches atteintes de la maladie, à constater l'abaissement de son taux au-dessous de $0 \mathrm{fr}$. 40 au litre ou tout au moins à constater ces oscillations de la glycémie dont nous avons parlé, bien que nous nous empressons de le dire, ces faits ne soient pas universellement admis, et à essayer le sérum glucosé dans le traitement de la maladie.

C'est ce qu'ont fait de nombreux auteurs en France et à l'étranger et nous devons dire tout de suite que les résultats qu'ils ont obtenu sont tout à fait différents. En France, G. et R. Moussu ont rencontré une glycémie à peu près normale dans 5 cas de 0,535 à $0,705 \%$ avec une moyenne de $0,646 \%$. En Angleterre, Maguire a trouvé une hypoglycémie dans les deux cas qu'il a examinés : 0,40 et $0,50 \%$. LitTLE et WRIGHT sur 24 cas ont rencontré tantôt une hypoglycémie, tantôt une glycémie normale, tantôt de l'hyperglycémie. En Amérique, HAYDEN et SHALL sont arrivés à des résultats identiques sur 14 vaches malades examinées : une fois, ils ont trouvé une glycémie remarquablement basse $0,35 \%$, d'autres fois un taux de suere normal, le plus souvent de l'hyperglycémie. WiDMark en Suède a pratiqué le dosage du sucre du sang sur 7 vaches atteintes de fièvre vitulaire; deux fois alors que la maladie débutait et paraissait bénigne, la glycémie était très basse : 0,36 et $0,33 \%$; dans les 5 autres cas il y avait nettement hyperglycémie de 3,80 à $1,12 \%$ de glucose. Alors que nous admettions que le syndrome hypoglycémique devait toujours s'accompagner d'une glycémie remarquablement basse, nous pensions comme WIDMARK et CARLENs que ces résultats ne devaient pas être pris en considération. Au moment où la fièvre vitulaire éclate, il ne faut point oublier que la mamelle est gonflée de lait et que la mulsion n'étant le plus souvent pas pratiquée, il y a rétention lactée. II s'en suit fatalement un passage du lactose dans le sang d'abord, dans les urines ensuite. Cette lactosurie est la règle dans la fièvre du lait; sur 15 urines de malades, PORCHER a trouvé 15 fois du lactose. Il s'en suit que lorsqu'on pratique le dosage du sucre du sang chez des femelles atteintes de fièvre vitulaire, on dose à la fois le glucose du sang et le lactose de rétention.

Jusqu'à ces derniers temps, on ne connaissait pas de méthode chimique permettant le dosage simultané du glucose et du lactose dans le sang. Des recherches encore inédites avaient été faites sur ce sujet dans le laboratoire du Professeur Ch. Porcher; maintenant nous le verrons plus loin cette lacune est comblée. Aussi, ne nous paraissait-il pas possible de tenir compte des chiffres trouvés dans les dosages de sucre du sang chez les femelles atteintes de fièvre vitulaire pratiqués tant en France qu'à l'Etranger et d'en tirer argument contre la théorie de l'hypoglycémie. On a rencontré, en effet, tantôt une quantité de sucre inférieure à la normale, tantôt une quantité normale, tantôt une quantité supérieure, 

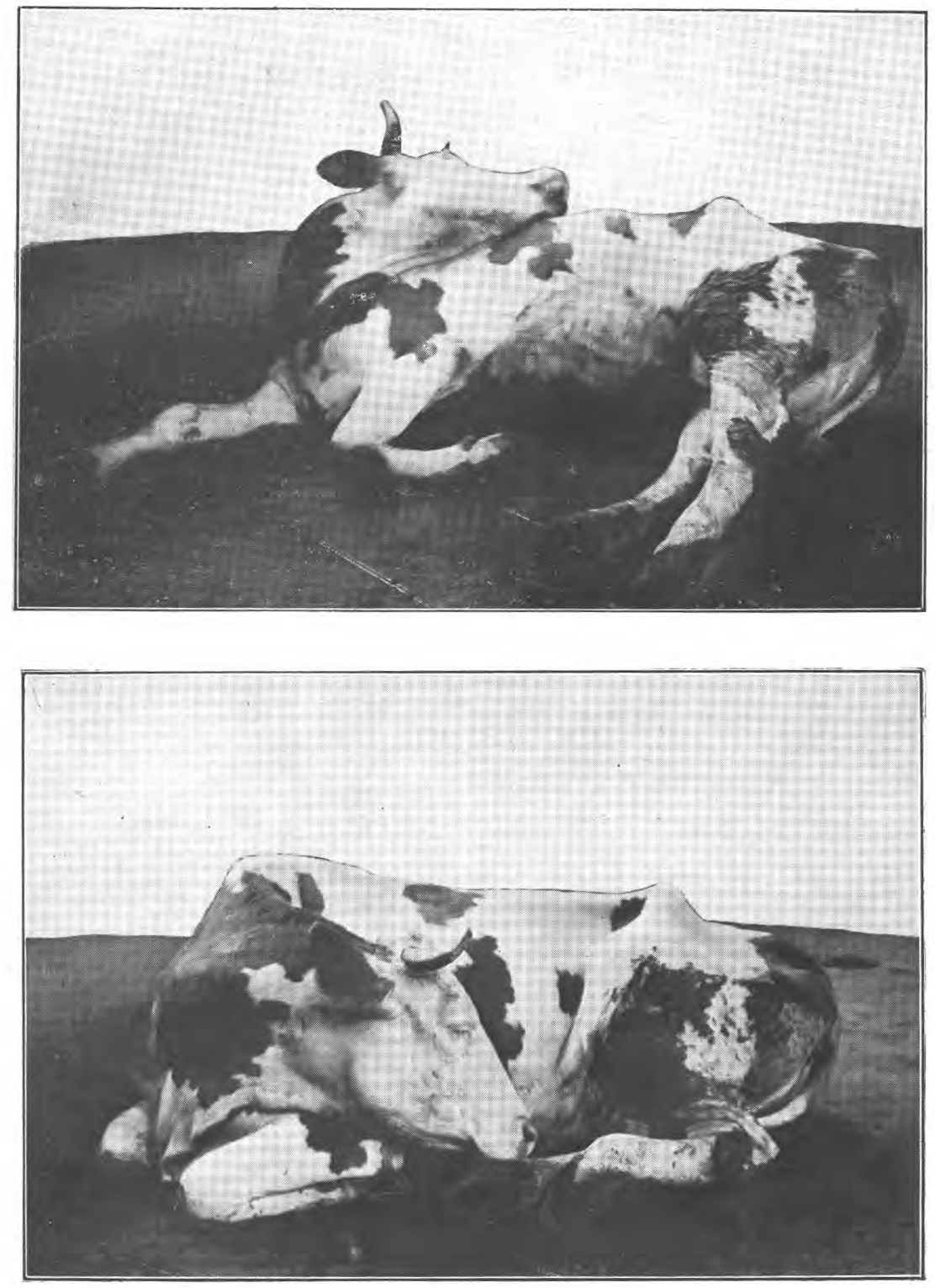

Deux attitudes du Coma hypo-glycémique. 

cela suivant què la rétention lactée était nulle, faible ou importante: à notre avis même étant donné la fréquence de la rétention lactée, l'hyperglycémie devait être la règle.

Nous ne pouvons plus faire état de ces arguments à l'heure actuelle. Deux auteurs américains, FouIN et SVEDBERG ont réussi à doser simultanément le lactose et le glucose du sang en utilisant la propriété que possède la levure de bière fraîche de faire fermenter seulement le glucose en respectant le lactose. Un autre expérimentateur américain, HAYDEN a immédiatement appliqué le procédé au dosage du sucre du sang de vaches atteintes de fièvre vitulaire. Il a vu que le lactose passait rarement dans le sang avant l'insufflation mammaire (une fois sur huit) et il a constaté que la quantité de glucose du sang était rarement inférieure à la normale (une fois), le plus souvent qu'elle était supérieure. Se basant sur les idées généralement admises que le syndrome hypoglycémique s'accompagne toujours d'une diminution du taux du glucose sanguin, que ce taux doit se maintenir uniformément bas pendant toute la durée des symptômes, il conclut que l'épuisement du glucose organique immédiatement utilisable ne doit pas intervenir au cours de la fièvre vitulaire, puisqu'on trouve fréquemment de l'hyperglycémie.

Nous répétons que de nombreux auteurs ont rencontré le syndrome hypoglycémique avee une glycémie normale ou même une hyperglycémie, que l'injection d'insuline n'agit pas seulement sur le glucose sanguin, mais sur les réserves hydrocarbonées de l'organisme qu'elle épuise et que pendant toute la dusée du syndrome hypoglycémique, la glycorégulation étant profondément troublée, le taux du sucre sanguin subit des oscillations souvent profondes allant de l'hypo- à l'hyperglycémie. Nous l'avons d'ailleurs démontré en injectant des doses considérables d'insuline à des animaux et en dosant le glucose sanguin toutes les trente minutes; nous avons constaté non pas une courbe uniformément basse, mais de très grandes oscillations. Il doit en être de même dans la fièvre vitulaire. Nous tenons à faire remarquer et nous insistons sur ce point, e'est que les auteurs qui ont publié des résultats du dosage du sucre du sang n'ont jámais fait qu'un seul prélèvement avant l'insufflation. Ils ont trouvé, suivant le moment, tantôt de l'hypoglycémie, tantôt une glycémie normale, tantôt de l'hyperglycémie, et ils en ont implicitement conclu que pendant tout le cours de la maladie, le taux du sucre trouvé devait rester constant. Or il est vraisemblable qu'avec des dosages effectués à des périodes variables du coma vitulaire, on trouverait ces oscillations de la glycémie qui paraissent constantes chez les animaux en coma insulinique, et peut-être à certains moments une glycémie remarquablement basse. La glycémie est une des constantes biologiques d'une importance extrême ; lorsqu'elle oscille au delà de certaines limites des troubles souvent graves apparaissent. En tenant compte des résultats obtenus par les auteurs eités plus haut, en les comparant, on voit 
qu'au eours de la maladie, cette glycémie varie dans des proportions considérables. HAYDEN en pratiquant des dosages du glucose sanguin après le traitement a toujours rencontré une augmentation nette de ce glucose qụi se maintient au dessus de la normale jusqu'à la guérison; il a confirmé ainsi les recherches de WIDMARK et CaRLENS et les nôtres sur l'effet du traitement d'Evers, il a vu aussi qu'après la guérison la glycémie revenait à son taux normal et s'y maintenait.

Il nous parait difficile de conclure, comme HAYDEN, que la glycémie, constante biologique d'une extrême importance, variant au cours d'une maladie, ees variations doivent être considérées comme négligeables; nous pensons au contraire qu'elles constituent toute la maladie, d'autant plus qu'elles cessent après la guérison.

II nous paraît utile de doser le glycogène du foje et des muscles chez les vaches mortes de fièvre vitulaire; il est vraisemblable qu'on le trouverait diminué en forte propostion.

En revanche, l'injection d'une solution glucosée influence favorablement l'évolution de la maladie. NeEF, WIDMaRk dans une conférence récente ont rapporté à ce sujet des faits probants et un vétérinaire anglais emploie indifféremment dans sa pratique l'injection glucosée ou l'insufflation mammaire avec des résultats aussi favorables. A la vérité, certains auteurs ont bien eu des échecs avec ce traitement. Nous pensons qu'il s'agit d'une question de doses. Il faut injecter une assez grande quantitéde glucose : 200 ou 300 grammes au moins, et faire l'injection dans les veines; sous la peau surtout, s'il s'agit de solutions hypertoniques, l'absorption est trop lente; les échecs sont dus à la trop petite quantité de glueose utilisée.

Reste à envisager la question du sucre dans l'urine. Dans ses études sur l'urologie de la fièvre vitulaire, Ch. Poroher a trouvé 15 fois sur 15 du lactose dans les urines et 6 fois du lactose et du glucose en petite quantité. Il semble bien à première vue du moins que la présence intermittente du glucose dans les urines soit un argument sérieux contre la théorie de l'hypoglycémie. S'il y a glycosurie véritable, c'est qu'il y a eu hyperglycémie. Nous avons examiné les observations de $\mathrm{Ch}$. PorCHER et toutes les fois que cet auteur a trouvé du glucose, nous avons pu nous convainere que l'urine avait été recueillie après l'insufflation des mamelles, celle-ci comme nous l'avons démontré relevant le taux du sucre du sang et pouvant provoquer l'hyperglycémie.

Il n'est done pas possible à l'heure actuelle de démontrer d'une façon directe qu'il y a hypoglycémie au cours de la fièvre vitulaire; il faut tenir compte cependant des effets de l'injection glucosée.

La fièvre vitulaire est une manifestation du syndrôme hypoglycémique ; c'est un trouble de la glyco-régulation, conséquence de la spécialisation zootechnique des animaux et parfois de lésions des organes qui règlent le métabolisme des hydrates de carbone. 
Il paraît indiscutable, ainsi que nous l'avons montré au début de ce travail, que la fièvre vitulaire est nettement conditionnée par le fonctionnement intense de la mamelle; si la mamelle demande beaucoup, c'est ce qui se passe chez une bonne laitière, il faut qu'elle pompe dans le sang le glucose, précurseur du lactose du lait, au point de déterminer parfois un abaissement notable de son taux dans le sang, et engendrer ainsi de l'hypoglycémie avec toutes les conséquences qui s'en suivent.

La fièvre vitulaire nous paraît done résulter, chez des animaux sélectionnés en vue de la production du lait, d'un manque momentané d'harmonie entre la mamelle en plein fonctionnement, d'une part, et les organes chargés de fournir au sang les eonstituants du lait, d'autre part, et plus particulièrement, puisqu'il s'agit de sucre ici, d'un manque de coordination entre les organes qui interviennent dans le métabolisme des hydrates de carbone, le foie en particulier, et la mamelle. La mamelle a besoin de beaucoup de glucose pour l'élaboration du lactose, le foie ne lui en fournit qu'une quantité insuffisante.

Si l'on se reporte au travail de Porcher sur l'origine du lactose, il est curieux de constater que cet auteur avait très bien mis en valeur, les relations du foie et de la mamelle, le foie organe glycogénétique la mamelle organe lactosogénitique, mais il n'avait signalé, dans la comparaison qu'il faisait du fonctionnement parallèle de ces deux glandes, que les cas où le foie donnait plu's que la mamelle n'exigeait, exemple la lactosurie ante-partum, post-partum, etc....

Il y a plus de 20 ans, quand il écrivait sur ces questions, l'étude de l'hypoglycémie n'occupait pas les chercheurs, comme elle le fait aujourd'hui. L'insuline était inconnue et la phloridzine n'offrait pas aux esprits le champ immense d'investigations que depuis lors nous a apporté l'insuline. C'est sans doute pour cette raison qu'il n'est pas venu à l'esprit dú Professeur Ch. Porcher, ainsi du reste qu'il prenait plaisir à nous le signaler lui-même, le cas où ce serait la mamelle qui demariderait au foie plus que celui-ci pourrait lui donner; aujourd'hui les études de l'insuline, en ce qui concerne le sujet traité dans ce travail, les recherches de Widmark et Carlens, de Maguire, les nôtres permettent de répondre que les troubles de coordination entre le foie et la mamelle peuvent done apparaître dans un sens ou dans un autre provoquant l'hyperglycémie dans un cas, l'hypoglycémie et par conséquent la fièvre vitulaire dans l'autre. L'organisme lutte facilement contre l'hyperglycémie, car lorsque le "seuil " est atteint, les émonctoires entrent en jeu, mais il paraît fort désarmé contre l'hypoglycémie. Si l'injection d'une dose massive de glucose est sans conséquence pour lui, il n'en est pas de même d'une dose massive d'insuline, et les animaux succombent.

Les oscillations à outrance que peut donner la production du lait peuvent bien amener ce déséquilibre momentané, cause de la fièvre 
vitulaire, et dues en somme à des exigences de la mamelle auxquelles le foie ne peut satisfaire.

R. GREIG insiste sur ce fait vraiment suggestif que la fièvre vitulaire n'a été connue qu'à partir de 1800 parce que, auparavant, les vaches n'étaient guère sélectionnées en vue de la production du lait.

Nous avons incidemment parlé tout à l'heure des relations possibles dans le cadre nosologique de la fièvre vitulaire de la vache et de l'éclampsie des autres espèces. Si la fièvre vitulaire est très souvent, le plus souvent même un simple trouble fonctionnel, conséquence d'une spécialisation très marquée cédant facilement au traitement devenu classique, on doit admettre cependant dans certains cas, notamment lorsqu'il existe des lésions des organes intervenant dans le métabolisme des hydrates de carbone, plus particulièrement du foie, que la maladie soit plus grave et dure plus longtemps. Ces lésions du foie, auxquelles se joignent fort souvent des lésions du rein, conditionnent donc la gravité de l'affection et ce sont ces faits qui nous permettent le rapprochement de la fièvre vitulaire avec l'éclampsie d'autres espèces.

A notre avis, on n'a pas tenu suffisamment compte dans l'examen étiogénique de l'hypoglycémie de la vache du rôle probable dévolu à la mise en train de la mamelle ; à force de ne considérer celle-ci que comme un phénomène des plus normaux, on n'a pas pensé que la glande va fonctionner intensément, qu'elle a des exigences alimentaires fort grandes qui peuvent engendrer des dysharmonies et des troubles.

En tous cas, nous pensons faire porter nos investigations sur l'éclampsie des autres espèces en étant guidé par les acquisitions des divers auteurs que nous a procurées l'étude de la fièvre vitulaire et sans doute trouverons-nous des faits intéressants.

Tout le premier, nous reconnaissons que la question de la fièvre vitulaire est loin d'être épuisée et qu'un grand nombre d'obscurités persiste encore, mais nous pouvons bien dire, en terminant, que la théorie de l'hypoglycémie, non pas simple spéculation de l'esprit, mais établie sur des bases expérimentales solides donne une explieation suffisante de la plupart des particularités de la maladie qui restait obscure avec les théories émises jusqu'à ce jour.

\section{BIBLIOGRAPHIE}

Auger L. Réalisation expérimentale de la fièvre vitulaire. Comptes-Rendus des séances de l'Académie des Sciences, 1926. page 348.

Adger L. Recherches sur la pathogénie de la fièvre vitulaire. Revue génér. de Méd.

Vét. 15 juillet 1926.

Auger L. Recherches sur la pathogénie de la fièvre vitulaire et le syndrome hypoglycémique chez la vache laitière. Thèse pour le Doctorat Vétérinaire. Lyon. 1926.

MagulRe. Etiology of Milk Fever. The Veterinary Record. 1926, p. 52.

Neef. L'insuline. Annales de Méd. Vét. 1923, p. 475.

Porcher Ch. L'origine du lactose, Archives Intern, de Physiol. 1909, p. 356. 
Poncher Ch. Contribution à l'étude de la lactosurie. Urologie de la fièvre vitulaire. Bullet. Soci. Centr. Méd. Vét. 1902, p. 409.

Póncher Ch. La rétention lactée. Archiv. de Méd. des Enfants. Octo.-Nov. 1920. Porcher $\mathrm{Ch}$. et Commandeur $\mathrm{Ch}$. Sur l'origine du lactose. Recherches urologiques chez la femme enceinte. Comptes-Rendus Acad. Scienc. 5 avril 1904.

Porcher et Leblanc. De la lactosurie chez les femelles pleines au moment du part. Bull. Soci. Centr. de Méd. Vét. 1902, p. 436.

Porcher et Panisset. Reeherches expérimentales sur le colostrum. Compt. Rend. Acad. Sci. 1921, p. 121.

Erick M. P. WIDMaRK und Olof CAelens. a) Ueber die Blutzuckerkonzentration bei Kuhen und den Einfluss der Lactationsintensität auf dieselbe. Biochemische Zeitschrift, t. CLVI, 1925, p. 454.

b) Durch Lufteinblasen in das Euter milehgebendener Tiere hervorgerufene Hyperglykämie. Id. t. CLVIII, 1925, p. 3.

c) Beobachtungen über die hypoglykämischen Symptome bei Kuhen. Id. t. CLVIII, 1925, p, 81.

Folin et Svedberg. Journal of biological Chimistry, 1906, p. 405.

HAYDEN. The Cornell Veterinarian. Avril 1927.

\section{BIBLIOGRAPHIE ANALYTIQUE}

\section{LES LIVRES}

Fascetti (G.). - II burro (Le beurre). 1 vol. relié, 152 p., 28 fig., 5 tables. G.B. Paravia, éd. Turin, 1927. Prix: lires 10,50.

Ce volume écrit avec l'habituelle clarté et la rigoureuse science du Professeur Fascetrt, appartient à la série zootechnique de la Bibliothèque agricole "Paravia ". Il traite de la fabrication rationnelle du beurre. Sa forme concise, qui répond au caractère de la collection à laquelle il appartient, est un grand mérite paree que

cet ouvrage est ainsi appelé à servir de guide non seulement aux élèves des cours de laiterie, mais également aux techniciens, aux contrôleurs et aux directeurs des beurreries, des laiteries et des erémeries, aux agriculteurs qui désirent faire du bon beurre eux-mêmes. C'est un véritable manuel de propagande beurrière s'appuyant sur les plus récentes théories expliquant le phénomène du barattage et qu'illustrent les plus récents systèmes mécaniques.

Le volume comprend, en outre de l'introduction qui parle de l'origine, de la production, du commerce et de la consommation du beurre, deux parties et un appendice.

La première partie traite de la matière première du beurre et après une étude bien conduite sur la graisse du lait (état physique, composition, propriétés physiques, influences quantitatives et qualitatives), FASCETTi trajte de l'écrémage naturel et de l'écrémage mécanique. Le phénomène de l'écrémage ainsi expliqué, la première partie se termine par un long chapitre consacré aux écrémeuses (structure, usage, inconvénients, contrôle).

La deuxième partie comprend l'étude du barattage. Elle passe en revue les divers principes théoriques invoqués pour l'expliquer, les machines nécessaires (barattes, malaxeurs, moules, etc.), ainsi que les règles pour pratiquer le barattage; elle se 